\title{
ZS Research Square

\section{Exploring the neural, behavioural, and clinical effects of transcranial direct current stimulation in patients with a Prolonged Disorder of Consciousness; protocol for a double-blind randomised crossover feasibility study}

Davinia Fernández-Espejo ( $\nabla$ d.fernandez-espejo@bham.ac.uk)

The University of Birmingham https://orcid.org/0000-0001-5941-7546

\section{Davide Aloi}

University of Birmingham

Antonio Incisa della Rocchetta

Wellington Hospital

\section{Damon Hoad}

University of Warwick Warwick Medical School

\section{Richard Greenwood}

Homerton University Hospital NHS Foundation Trust

E Diane Playford

University of Warwick Warwick Medical School

\section{Damian Cruse}

University of Birmingham

\section{Study Protocol}

Keywords: Disorder of consciousness, minimally conscious state, vegetative state, magnetic resonance imaging, transcranial direct current stimulation, rehabilitation

Posted Date: February 28th, 2020

DOI: https://doi.org/10.21203/rs.3.rs-15515/v1

License: (c) (1) This work is licensed under a Creative Commons Attribution 4.0 International License.

Read Full License 


\section{Abstract}

Background: Therapeutic options for patients with prolonged disorders of consciousness (PDOC) are very limited, and patients often show little to no progress over time. It is widely recognized that some PDOC patients retain a higher level of cognition that may be apparent on the basis of their external responses, and simply are unable to produce purposeful motor behaviours. This dissociation has been linked to specific impairments in the motor network that lead to a reduction in thalamo-cortical coupling. Here, we will assess whether transcranial direct current stimulation (tDCS) can modulate thalamo-cortical coupling and improve patients' responsiveness. We will focus on characterising the mechanisms of action of tDCS and the bases for potential individual differences in responsiveness to the stimulation across participants.

Methods: This is a multi-centre double-blind randomised crossover feasibility study. It is divided into two streams: (a) MRI stream: 5 PDOC patients will complete 5 anodal, cathodal, and sham stimulation sessions (paired with passive mobilisation of the thumb) in separate weeks. We will measure brain activity and connectivity with functional magnetic resonance imaging and electroencephalography (EEG). We will look at brain structures to assess differences associated with responsiveness. (b) Bedside stream: 10 patients will complete one session of anodal or cathodal stimulation and one session of sham. We will measure brain activity and connectivity with EEG and we will conduct follow up assessments at 3 and 6 months.

In both streams we will also look at changes in the clinical profile of patients with the Coma Recovery Scale Revised and in command following behaviour with electromyography and motion tracking.

We will assess feasibility on measures of eligibility, recruitment, retention, and completion of tests.

Discussion: This feasibility study is the first step towards developing personalised tDCS interventions to restore external responsiveness in PDOC patients. Our results will inform the design of a future trial fully powered for characterising neural, behavioural, and clinical effects of tDCS in PDOC as well as the mechanisms underlying individual differences in responsiveness.

\section{Background}

\section{Background and rationale}

Prolonged disorders of consciousness (PDOC), such as the vegetative state (VS) or the minimally conscious state (MCS), lead to catastrophic disability and complex needs for care and treatment [1]. Prognosis is poor and diminishes over time [2], with a high proportion of patients surviving for many years with little to no functional improvement [3]. Alongside a high psychosocial cost for families [4], the cost of PDOC care is estimated at $£ 92 \mathrm{k}$ per patient per year [5]. However, no therapies have proven successful for inducing or accelerating recovery. VS patients show no overt behavioural signs of awareness and are considered to be entirely unaware. In contrast, MCS patients show reproducible signs 
of awareness but these are typically inconsistent and fluctuate greatly across assessments. In a first step towards identifying successful interventions, we recently demonstrated that specific impairments in structural connectivity within the motor system can result in reduced excitatory coupling between thalamus and the primary motor cortex (M1), which in turn leads to reduced (or absent) external responsiveness in PDOC patients [6, 7]. Importantly, the damage varies alongside the severity of the clinical condition but it remains partial, as opposed to a complete deafferentation, indicating that the function of the remaining pathways may be amenable to therapeutic strengthening. On this basis, we hypothesise that interventions to enhance the flow of information between thalamus and M1 will provide patients with a renewed level of control over their external behaviour.

One prevailing approach for modulating brain function is transcranial direct current stimulation (tDCS) [8], which alters neural excitability in a polarity-specific manner via a weak electric current delivered through electrodes on the scalp [9]. tDCS has been shown to affect functional cortico-thalamic networks in healthy volunteers [10-13] and neurologically impaired patients [14, 15]. However, for the neural effects to translate into reliable changes in motor behaviour, tDCS must be paired with concurrent motor training over multiple stimulation sessions [16-18]. PDOC patients are, by definition, incapable of engaging in voluntary motor training. Nevertheless, these patients receive regular passive mobilisation as maintenance therapy to prevent the development of contracture [1]. Moreover, passive mobilisation of the upper limbs results in similar fMRI activity as compared to active, voluntary movement in both healthy volunteers and VS patients [19]. Indeed, passive movements have been related to changes in corticomotor excitability $[20,21]$, and are regularly used in rehabilitation programmes for other neurological groups [22]. Therefore, the evidence indicates that passive movement, combined with tDCS, may cause similar augmentative effects to those observed with active movements. Evidence from stroke further supports this conclusion [23].

To date, sixteen studies have used tDCS in PDOC without adverse effects [24-29]. These reported inconsistent changes in cortical excitability $[26,27]$ and transient improvements in signs of consciousness $[24,25,28,29]$ in a small percentage of MCS patients. While these results represent an important proof-of-principle, the selection of target regions (e.g., M1 vs. dorsolateral prefrontal cortex), electrode montage, and intensity/duration of stimulation was highly heterogeneous across studies. Moreover, many lacked adequate control conditions (e.g. anodal/cathodal/sham) and/or failed to account for individual differences in structural integrity of the brain and the fluctuations in behaviour that characterise many PDOC patients [30]. These limitations make the interpretation of their outcomes very challenging and preclude making specific recommendations about which stimulation parameters are the most effective. In fact, the neural mechanisms underlying tDCS related changes both in the healthy and injured brain are still very poorly understood [8]. Here, we propose a multimodal neuroimaging and electrophysiology study to characterise such mechanisms in PDOC. To our knowledge, this is the first study combining functional and structural magnetic resonance imaging (MRI) and electrophysiology methods to study the effects of tDCS both online (during stimulation) and offline (after stimulation).

\section{Aims}




\section{Primary}

1. To test the feasibility of an experimental protocol that combines advanced multi-modal imaging of the brain (MRI, EEG) with clinical (Coma Recovery Scale) and overt and subthreshold motor responses (motion capture, EMG) to characterise the neural, behavioural, and clinical effects of tDCS for rehabilitation in PDOC. We will quantify eligibility and recruitment rates, percentage of participants still active at different stages of the study and percentage of tests completed. We will also review records for:

- Willingness of potential participants' personal or nominated consultee to take part

- Potential barriers to recruitment

- Potential barriers to retention and completion of the study

\section{Secondary}

1. To explore the effects of tDCS coupled with passive mobilisation in brain activity, functional and structural connectivity of the motor network and external responsiveness (motor function and clinical assessments) in PDOC patients

2. To explore the relationship between structural and functional integrity of each patient's motor network and their individual responses to stimulation.

\section{Study design}

This is a double-blind randomised crossover feasibility study of tDCS in a small cohort of PDOC individuals. The study is divided into two streams to accommodate differences in the resources available across recruitment sites (see Study Flowchart in Figure 1):

\section{MRI stream}

We will recruit a convenience sample of 5 patients at a specialised private rehabilitation unit with in house MRI facilities. All participants will complete a double-blind crossover protocol of anodal, cathodal, and sham (placebo) stimulation in a randomised order, to fully control for confounds and allow for confident conclusions [8] (Figure 1). This will include 5 daily sessions of each stimulation condition (anodal, cathodal, sham). Each group of 5 sessions will include 2 sessions in the MRI scanner (day 1 and day 5) and 3 at bedside (days 2-4) paired with electrophysiological recordings. Additionally, we will capture the clinical status of each participant before and after stimulation in all 5 sessions with the current clinical diagnostic standard (CRS-R [31]). Each group of five sessions will be separated by a minimum of 7 days to allow for wash out [32].

\section{Bedside stream}

To assess feasibility, we will recruit a convenience sample of 10 patients across multiple rehabilitation sites (including NHS and non-NHS). We are able to recruit a higher number of patients in this stream as it 
involves fewer resources. Specifically, recruitment sites in this stream do not have access to MRI facilities. Participants will receive a double-blind crossover protocol of sham-controlled tDCS, in which polarity is controlled cross-sectionally, due to their lower time available for research at these sites.

Specifically, each participant will receive one session of active tDCS (anodal or cathodal) and one session of sham separated by a minimum of 7 days. Each session will be paired with electrophysiological and clinical (CRS-R) assessments. Additionally, as patients at this rehabilitation recruitment sites can be more easily tracked after their discharge (compared with those in the MRI stream), we will use this opportunity to also collect follow-up data at 3-months and 6-months post tDCS sessions with the Glasgow Outcome Score Extended (GOSE) [33], a standard clinical outcome assessment tool.

Insert Figure 1 here.

\section{Methods}

\section{Participants, interventions and outcomes}

\section{Study setting}

For the MRI stream we will recruit participants from a cohort of PDOC patients who are receiving care at the PDOC unit (PDOCU), at the Wellington Hospital, HCA Healthcare. All study assessments (including MRI) will take place in the hospital facilities, making it an ideal location to conduct this complex research with minimal patient burden. On average, patients stay in this unit for 4 months, which will facilitate the longitudinal approach for this stream.

For the bedside stream we will recruit participants from available cohorts of PDOC patients receiving care at specialised units in NHS and non-NHS sites. Typically patients stay in these units for approximately 10 weeks.

\section{Eligibility criteria}

To be included in this study, the participant should be:

1. Aged 18 years or older

2. Receiving care at a recruitment site, with a consensus clinical diagnosis of PDOC from any aetiology (i.e. traumatic or non-traumatic injury).

3. Stable and with no need of mechanical support (i.e. respirator, etc.)

The presence of any of the following will preclude participant inclusion:

1. Scalp skin sores or any skin damage at the electrode sites

2. Metallic implants in the face or skull

3. Craniectomy or cranioplasty 
4. No evidence of auditory startle in clinical observations, or absent brainstem auditory evoked potentials in recent clinical history (if data available)

5. MRI incompatible: metal plates incompatible with MRI scanners, pacemaker, inability to lay flat for prolonged periods of time, aneurysm clips, neurostimulators, brain/subdural electrodes, etc. (MRI stream ONLY)

\section{Recruitment, consent, and assignment of interventions}

A clinical member of the research team at each recruiting centre will screen participants for their eligibility for inclusion into the study, identify a personal consultee (or nominated consultee if a personal consultee is not available), and make the first approach to inform them about the study. If the consultee is interested, they can provide consent to be contacted by the research team who will then directly contact them to provide the letter of information and complete the consent process. The consent process will be performed in compliance with the Mental Capacity Act [34]. We will give the consultees 1 week to consider participation and to discuss their participation with others outside of the site research team. The consultee will be given the opportunity to ask questions before signing and dating the consent forms.

Their willingness to continue in the study will be ascertained and documented throughout the study. If the consultee decides to withdraw the patient's participation in the study, they can inform any member of the research team at any time and data collection will stop immediately. The Research Team will retain the data already collected, unless the consultee requests the withdrawal of the patient's data too. In this case, the Research Team will remove all identifiable data any de-identified data collected up to 2 weeks prior to obtaining the withdrawal notification.

The suitability of each participant for MRI and tDCS testing will be determined by a clinical member of the research team in collaboration with the patient's main Consultant. Therefore, all eligible participants will be screened for contraindications to MRI (MRI stream only) and tDCS (both streams). The research team will re-screen the patient before each subsequent MRI and tDCS sessions, and consult with the clinical team if any new information arises.

At enrolment, the research team will use in-house computer code to randomly select the specific order for the participant amongst the following possible combinations, ensuring a balanced distribution of participants across them:

- MRI stream:

1. Anodal, cathodal, sham

2. Anodal, sham, cathodal

3. Cathodal, anodal, sham

4. Cathodal, sham, anodal

5. Sham, anodal, cathodal

6. Sham, cathodal, anodal 
- Bedside stream:

1. Anodal, sham

2. Cathodal, sham

3. Sham, anodal

4. Sham, cathodal

We will not start testing until the patient is clinically stable and some preliminary diagnostic assessments have been performed by the clinical team to confirm the patient is indeed in a PDOC.

\section{Recovery of consciousness}

The clinical members of the research team will regularly assess participants' clinical diagnosis and capacity throughout the study. If any participant exits the clinical definition of PDOC (i.e., moves beyond a diagnosis of emerging from the minimally conscious state) and / or regains capacity, their data will be kept but their participation in the study will be ended (i.e., they will not receive any further sessions of stimulation or imaging).

\section{tDCS intervention}

We will use the following commercially available tDCS stimulators, manufactured and sold by neuroCare Group GmbH: neuroConn DC-STIMULATOR and/or DC-STIMULATOR PLUS (both for the sessions outside the MRI scanner), and neuroConn DC-STIMULATOR MR (MRI compatible version of the DC-STIMULATOR PLUS) for the sessions in the MRI scanner. All three systems have EU Declarations of Conformity with the following standards or normative documents, including those for medical electrical equipment, and medical devices: EN60601-1:2006+A1:2013; EN60601-1-2:2007; EN60601-1-6:2010+A1:2015; EN62304:2006; EN62366:2008+A1:2015; EN ISO 10993-1:2009; EN ISO 14971:2012;

Each stimulation session will consist of 20 minutes of a maximum of 2mA tDCS over the left M1 (return electrode over the right frontal pole). We will pair the stimulation with slow passive mobilisation of the right thumb to engage the motor network and enhance the effects of tDCS. The protocol for stimulation has been designed in collaboration with the physiotherapy team at the PDOCU to ensure patient safety.

If clinical notes and scans show evidence of severe macrostructural structural damage to the left motor strip at recruitment for any given patient, we will apply tDCS to the right hemisphere and passive mobilisation to the left thumb.

\section{Blinding}

The study will be double-blind (participants and researchers). We will keep information about the specific stimulation condition delivered in each session in the study file and accessible to the Chief Investigator $(\mathrm{Cl})$ only until data analyses have been completed. If any adverse events (AE) are suspected, the $\mathrm{Cl}$ will make this information available to the participant's clinical team. A consultant who is both part of the 
research team and the care team will decide whether it is safe for the patient to continue their participation in the study. If deemed safe by the consultant, unblinded participants will be allowed to continue to participate in the study. In such case, double-blinding will be implemented in subsequent sessions, and for the analyses of the data.

\section{Outcome assessments}

This study is exploratory in nature and therefore the outcome measures include an array of neuroimaging, electrophysiological and clinical measures that will be used to inform future larger trials.

1. MRI (MRI stream only): participants will undergo a total of $6 \mathrm{MRI}$ sessions (2 per stimulation condition), including assessments of the macro and microstructure of grey matter, white matter, and cerebrospinal fluid (T1, T2, and diffusion weighted imaging); the baseline and post tDCS brain activity and connectivity at rest and during command following; and brain activity during the administration of tDCS.

2. Electrophysiology (both streams): participants will receive 9 assessments in the MRI stream (3 per stimulation condition), and 2 assessments in the bedside stream (1 per stimulation condition). These will include resting state EEG as well as a recording of the activity across the motor pathway in response to commands to move - from sensorimotor cortex $(\operatorname{EEG}[35,36])$ to peripheral neurons in the arm (EMG [37, 38]) to sub- and supra-threshold movements (motion tracking).

3. Baseline and post-tDCS CRS-R scores on each stimulation session (both streams).

4. Outcome at 3 and 6 months with GOSE (bedside stream only), via follow-up telephone calls with the participant or their consultee.

See Table 1 for the schedule of assessments in each stream

Insert Table 1 here.

\section{Data management and monitoring}

All participants will be assigned a unique identifier (e.g. p001) upon recruitment. Identifiable data will be stored alongside this identifier, and kept secure in locked filing cabinets and password-protected servers at the Wellington Hospital and the University of Birmingham.

We will record consultee's contact details as well as details about each participant clinical journey on an electronic Case Report Form (CRF). MRI data will be downloaded from the scanner into a passwordprotected encrypted portable hard drive or burnt into a DVD by the hospital's staff, after being anonymised. EEG, EMG, and motion tracking data will be recorded in dedicated password protected laptops registered with the University of Birmingham. CRS-R and GOSE data will be collected on paper forms and recorded in the electronic CRF. 
Data will be transferred to the secure user accounts at the University of Birmingham to enable further processing and analyses using co-investigators. All data will be stored at UOB for 10-years. The Chief Investigator is the data custodian. Identifiable data will be stored separately from de-identified data in physically separate locked filing cabinets and separate password-protected server directories. Access to data will be limited by the $\mathrm{Cl}$ to those necessary (e.g., the Research Team, Sponsor, and regulatory authorities) for quality control, audit, and analysis, to ensure security.

All investigators and study site staff will comply with the requirements of the General Data Protection Regulation (GDPR) / Data Protection Act 2018 with regards to the collection, storage, processing and disclosure of personal information and will uphold the Act's core principles. No documents will be destroyed without prior approval from the Chief Investigator.

\section{Data analysis}

\section{MRI and Electrophysiology}

Our sample size does not offer enough power for a full characterisation of our secondary aims. Instead our analyses will be exploratory and allow us to identify indices and analysis pipelines for the subsequent fully powered study. We will assess tDCS-induced changes in brain activity as well as functional and effective connectivity across the motor network at rest and during simple command following using fMRI and EEG. We will then link potential changes in these variables with the changes in behaviour (motion tracking), peripheral activity (EMG), and clinical status (CRS-R).

To investigate the relationship between patient-specific preservation of neural architecture and responsiveness to our stimulation protocol we will assess a number of quantitative structural metrics including diffusion weighted indices (e.g., fractional anisotropy of key white matter tracts), or volume and thickness of cortical and subcortical structures, amongst others $[39,40]$. Additionally, we will characterise participant's baseline functional architecture with EEG and fMRI. We will assess whether any of these measures can predict individual responses to tDCS. We will quantify EEG measures of functional architecture pre- and post-tDCS as per [41] and will investigate task-related EEG changes in the mu and beta bands [35].

\section{Feasibility data}

We will record data about eligibility, recruitment, retention, and completion of tests, and report summary statistics. We will also record qualitative information about potential barriers to any of the above. The small sample size in this study may not allow for an accurate estimation of recruitment and retention rates. However, we suggest that our protocol is feasible if we achieve the following retention and completion rates:

- MRI stream: $65 \%$ of participants complete at least 4 sessions of tDCS per polarity including at least 1 in the MRI per polarity. They also complete at least 1 full EEG assessment per polarity. 
- Bedside stream: $65 \%$ of participants complete both tDCS sessions (including the corresponding EEG assessments). $75 \%$ of participants are still available at 3-month follow-up and $65 \%$ at 6 -months.

If we are not able to meet the above figures we will consider adjusting the protocol for future studies. Insert Table 2 here.

\section{Safety}

\section{MRI}

There are no known risks of undergoing an MRI examination 3T scanner per se. The procedure is considered to be completely safe and non-invasive. MRI has now been extensively used for over 20 years for diagnostic radiology, and widely used for over 10 years in research laboratories. MRI is also routinely used at the Wellington Hospital as part of their clinical service. The clinical team at the Wellington Hospital will screen participants for MRI safety following local SOP, and will approve the transport to the patient to the MRI suite for each session.

All researchers involved in the scanning of participants will have participated in an MRI safety course. The patient transfer from the ward to the imaging suite, and the scanning session will be performed by qualified members of staff at the Wellington Hospital and will be performed according to local clinical SOP.

Some of the sequences in our protocol (e.g., anatomical data) have clinical value and therefore will be made available to the patient's clinical team for review.

\section{tDCS}

When used in accordance with specified and long established guidelines [42, 43], tDCS poses a negligible risk to participants. The most commonly reported effects are: a tingling or burning sensation under the electrodes that typically subsides after a few seconds; fatigue; and mild headaches that respond well to over-the-counter painkillers [43]. Moderate adverse events (AE) are rare and include: skin lesions (e.g., burns), which are typically related to pre-existing conditions or suboptimal electrode-skin contact; and very rarely mania or hypomania in patients with depression [43].

We will minimise these effects by screening participants for skin conditions on or around the areas where electrodes will be applied and their current medication. Additionally, the effects of tDCS in patients with brain or skull metallic implants are not well studied. While the intensity of the current is unlikely to produce heating, current induction, or movement, we will also screen for implants and exclude participants accordingly (see exclusion criteria). If there are any concerns as to whether it is safe for the patient to participate in the study, the tDCS operator will seek advice from the PI and / or the medical team. If there is any uncertainty concerning safety, the participant will be excluded. 
Only experienced qualified researchers will administer tDCS to the participants. They will make sure that skin and stimulation electrodes are prepared according to guidelines $[43,44]$ and will monitor signs of discomfort at all times. Should any discomfort appear, the stimulation will be terminated immediately.

It is worth noting that there are 16 published studies of tDCS with similar parameters to those in our study, totalling over 200 PDOC patients and no adverse effects reported [45]. In a recent study, Martens and colleagues delivered 20 sessions of 20 minutes $2 \mathrm{~mA}$ tDCS to a group of 27 patients and reported no tDCS-related adverse effects other than mild skin redness or sleepiness in $1 \%$ of the sessions [46].

\section{tDCS in the MRI}

There are no reported risks related to the delivery of the tDCS in the scanner, other than those with tDCS alone, when the correct equipment is used (i.e., MRI compatible tDCS system, biocarbon electrodes, and electrical conductive paste).

\section{EEG}

EEG is a common procedure in clinical care and has no risks for participants.

\section{Adverse Events}

We do not anticipate any serious adverse events (SAEs) related to any of our methods. However, we will monitor the safety of our protocol and record all adverse events (AE) in the electronic CRF. Clinical members of the research team will assess seriousness, causality (relatedness), an expectedness of any AEs. The $\mathrm{Cl}$ will report any SAE that suggests causality and unexpectedness will be reported to the Sponsor and REC within 15 days of becoming aware. If the clinician responsible for the care of the participant deems that it is clinically necessary to stop their participation in the study, we will withdraw them. However, we will still invite the participant to complete the follow up assessments (phone interview with next of kin).

\section{Discussion}

This feasibility study is the first step towards developing personalised tDCS interventions to restore external responsiveness in PDOC patients. Our protocol includes a series of advanced multimodal neuroimaging and electrophysiology methods to assess specific neural changes during and after tDCS in each individual patient. This rich data will provide us with a snapshot of the mechanisms of action explaining potential tDCS-induced changes in our participants' external behaviour and/or clinical status. Importantly, for some patients, our methods may detect potential subthreshold changes in neural activity that are not yet strong enough to lead to reliable behavioural responses, and would call for future adjustments in the protocol.

We will also investigate how individual differences in the preservation (or otherwise) of key structural and functional networks can predict individual responses to specific stimulation parameters. Previous 
research suggests that, even in healthy participants, subtle differences in neuroanatomy may explain up to $50 \%$ of interindividual variability in the electric field generated by tDCS $[47,48]$. It can only be expected that this effect is more pronounced in PDOC patients, who typically present severe and widespread brain atrophy and a high heterogeneity in the individual patterns of damage [49]. Our data may thus aid in the development of stimulation programmes that are personalised to each individual's relative neural preservation. Our study has not been powered for hypothesis testing, but our data will inform the design and delivery of future fully powered studies to fully explore these aspects. The ultimate goal of this study is to assess whether our complex design is feasible in a clinical setting and to establish the infrastructure for a larger study if feasibility is confirmed.

If our methods are successful, the increased behavioural responsiveness will allow some patients to use assistive devices to regain a level of autonomy for the first time since their injury. Importantly, even if only transient improvements are achieved, these will open the door for patients to actively participate in some motor/cognitive rehabilitation programmes (e.g. physical therapy, neuropsychology, speech and language therapy), which are not currently indicated for PDOC [1]. Indeed, active rehabilitation is associated with accelerated recovery and shorter hospital stays [50]. Furthermore, increased patient autonomy and reduced hospitalisation time will greatly reduce the healthcare costs of this challenging population. Importantly, our work to date suggests that motor impairments also interfere with patients' ability to demonstrate their 'true' cognitive functioning. While PDOC patients are considered to be entirely or partially unconscious (VS and MCS, respectively), some patients retain a significantly higher level of cognitive function than is evident from their behaviour. Specifically, functional neuroimaging has revealed that $15-20 \%$ of VS patients are in fact aware but simply unable to exhibit overt purposeful responses [51, 52]. For example, in one patient who had been repeatedly diagnosed as VS for 12 years, we successfully used fMRI to demonstrate that he was not only aware, but also able to form memories and communicate answers to clinically relevant questions [51]. In this context, providing patients with a degree of control over their external behaviour will have profound implications not only for quality of life in those who are aware, but also for accuracy of diagnoses across all patients. There is a chance that our imaging tests reveal signs of covert cognition in some of our participants. If this occurs, we will feedback this information to the participant's clinical team to be communicated to them and their families following PDOC ethical guidelines [53].

To our knowledge, this is the first study to combine structural and functional MRI with electrophysiology to characterise the neural effects of tDCS in PDOC from a specific mechanistic hypothesis to explain their lack of voluntary external responses. Our long term goal is to acquire a deep understanding of the mechanisms underlying the effects of tDCS in this patient group and differences across patient's responses. This will allow us to design more effective protocols that can be tailored to the individual needs of patients.

\section{Abbreviations}


AE:Adverse Event; Cl:Chief Investigator; CRS-R:Coma Recovery Scale-Revised;

EEG:Electroencephalography; EMG:Electromyography; fMRI:Functional Magnetic Resonance Imaging; GDPR:General Data Protection Regulation; M1:Primary motor cortex; mA:Milliamps; MCS:Minimally Conscious State; MRI:Magnetic Resonance Imaging; PDOC:Prolonged Disorders of Consciousness; PDCOCU:Prolonged Disorders of Consciousness Unit (at the Wellington Hospital); SFTP:Secure File Transfer Protocol; SOP:Standard Operating Procedure; T:Tesla; tDCS:Transcranial Direct Current Stimulation; UoB:University of Birmingham; VS:Vegetative State.

\section{Declarations}

\section{Ethics approval}

The West Midlands - Coventry and Warwickshire Research Ethics Committee provided ethical approval for this study (19/WM/0180).

\section{Sponsor information}

The University of Birmingham provided Sponsorship for this study (RG_18-269). We drafted the manuscript according to version 4.0 (10th February 2020) of the protocol.

\section{Acknowledgements}

The authors would like to thank Sara Assecondi for her feedback on early versions of the protocol.

\section{Funding}

The Wellington Hospital provided in-kind support for this study and co-funded a PhD scholarship (DA). The College of Life and Environmental Sciences, and the School of Psychology, University of Birmingham, further supported the study and co-funded DA's PhD. The University of Birmingham and the Wellington Hospital as sponsors and funders have not had any input into the study design and will not have a role in data collection or analysis. Aspects of this research are further supported by MRC-NIRG (MR/P02596X/1; DF-E) and MRC-NIRG (MR/P013228/1; DC).

\section{Availability of data and materials}

Fully anonymised data can be made available to other researchers upon request to the Chief investigator

\section{Authors' contributions}

DFE, Chief Investigator, developed the initial project idea and study design and led on the writing of the manuscript. DA, DFE, Al, and DC contributed to the study design, set-up, management, and data collection. RG, DP, and DH provide clinical support to the study. All authors revised and approved the final manuscript.

\section{Competing interests}


The authors declare that they have no competing interests.

\section{Consent for publication}

Not applicable

\section{References}

1. Royal College of Physicians of London. Prolonged disorders of consciousness: national clinical guidelines. 2013.

2. The Multi-Society Task Force on PVS. Medical aspects of the persistent vegetative state (I). 1994.

3. Estraneo A, Moretta P, Loreto V, Lanzillo B, Santoro L, Trojano L. Late recovery after traumatic, anoxic, or hemorrhagic long-lasting vegetative state. Neurology. 2010;75:239-45.

4. Covelli V, Sattin D, Giovannetti AM, Scaratti C, Willems M, Leonardi M. Caregiver's burden in disorders of consciousness: a longitudinal study. Acta Neurol Scand. 2016;134:352-9.

5. Formby A, Cookson R, Halliday S. Cost Analysis of the Legal Declaratory Relief Requirement for Withdrawing Clinically Assisted Nutrition and Hydration (CANH) from Patients in the Permanent Vegetative State (PVS) in England and Wales. 2015;:23.

6. Fernández-Espejo D, Rossit S, Owen AM. A Thalamocortical Mechanism for the Absence of Overt Motor Behavior in Covertly Aware Patients. JAMA Neurol. 2015;72:1442.

7. Stafford CA, Owen AM, Fernández-Espejo D. The neural basis of external responsiveness in prolonged disorders of consciousness. Neurolmage Clin. 2019;22:101791.

8. Parkin BL, Ekhtiari H, Walsh VF. Non-invasive Human Brain Stimulation in Cognitive Neuroscience: A Primer. Neuron. 2015;87:932-45.

9. Nitsche MA, Paulus W. Excitability changes induced in the human motor cortex by weak transcranial direct current stimulation. J Physiol. 2000;527:633-9.

10. Lang N, Siebner HR, Ward NS, Lee L, Nitsche MA, Paulus W, et al. How does transcranial DC stimulation of the primary motor cortex alter regional neuronal activity in the human brain?: tDCS-induced changes of rCBF. Eur J Neurosci. 2005;22:495-504.

11. Polanía R, Paulus W, Nitsche MA. Modulating cortico-striatal and thalamo-cortical functional connectivity with transcranial direct current stimulation. Hum Brain Mapp. 2012;33:2499-508.

12. Sankarasubramanian V, Cunningham DA, Potter-Baker KA, Beall EB, Roelle SM, Varnerin NM, et al. Transcranial Direct Current Stimulation Targeting Primary Motor Versus Dorsolateral Prefrontal Cortices: Proof-of-Concept Study Investigating Functional Connectivity of Thalamocortical Networks Specific to Sensory-Affective Information Processing. Brain Connect. 2017;7:182-96.

13. Sours C, Alon G, Roys S, Gullapalli RP. Modulation of Resting State Functional Connectivity of the Motor Network by Transcranial Pulsed Current Stimulation. Brain Connect. 2014;4:157-65.

14. Antal A, Terney D, Kühnl S, Paulus W. Anodal Transcranial Direct Current Stimulation of the Motor Cortex Ameliorates Chronic Pain and Reduces Short Intracortical Inhibition. J Pain Symptom Manage. 2010;39:890-903.

15. Benninger DH, Lomarev M, Lopez G, Wassermann EM, Li X, Considine E, et al. Transcranial direct 
current stimulation for the treatment of Parkinson's disease. J Neurol Neurosurg Psychiatry. 2010;81:1105-11.

16. Bolognini N, Pascual-Leone A, Fregni F. Using non-invasive brain stimulation to augment motor training-induced plasticity. J NeuroEngineering Rehabil. 2009;6:8.

17. Hashemirad F, Zoghi M, Fitzgerald PB, Jaberzadeh S. The effect of anodal transcranial direct current stimulation on motor sequence learning in healthy individuals: A systematic review and meta-analysis. Brain Cogn. 2016;102:1-12.

18. Orban de Xivry J-J, Shadmehr R. Electrifying the motor engram: effects of tDCS on motor learning and control. Exp Brain Res. 2014;232:3379-95.

19. Weiller C, Jüptner M, Fellows S, Rijntjes M, Leonhardt G, Kiebel S, et al. Brain Representation of Active and Passive Movements. Neurolmage. 1996;4:105-10.

20. Chye L, Nosaka K, Murray L, Edwards D, Thickbroom G. Corticomotor excitability of wrist flexor and extensor muscles during active and passive movement. Hum Mov Sci. 2010;29:494-501.

21. Lewis GN, Byblow WD, Carson RG. Phasic modulation of corticomotor excitability during passive movement of the upper limb: effects of movement frequency and muscle specificity. Brain Res. 2001;900:282-94.

22. Volpe BT, Ferraro M, Lynch D, Christos P, Krol J, Trudell C, et al. Robotics and other devices in the treatment of patients recovering from stroke. :6.

23. Edwards DJ, Krebs HI, Rykman A, Zipse J, Thickbroom GW, Mastaglia FL, et al. Raised corticomotor excitability of M1 forearm area following anodal tDCS is sustained during robotic wrist therapy in chronic stroke. Restor Neurol Neurosci. 2009;27:199-207.

24. Angelakis E, Liouta E, Andreadis N, Korfias S, Ktonas P, Stranjalis G, et al. Transcranial Direct Current Stimulation Effects in Disorders of Consciousness. Arch Phys Med Rehabil. 2014;95:283-9.

25. Huang W, Wannez S, Fregni F, Hu X, Jing S, Martens G, et al. Repeated stimulation of the posterior parietal cortex in patients in minimally conscious state: A sham-controlled randomized clinical trial. Brain Stimulat. 2017;10:718-20.

26. Naro A, Calabrò RS, Russo M, Leo A, Pollicino P, Quartarone A, et al. Can transcranial direct current stimulation be useful in differentiating unresponsive wakefulness syndrome from minimally conscious state patients? Restor Neurol Neurosci. 2015;33:159-76.

27. Naro A, Russo M, Leo A, Cannavò A, Manuli A, Bramanti A, et al. Cortical connectivity modulation induced by cerebellar oscillatory transcranial direct current stimulation in patients with chronic disorders of consciousness: A marker of covert cognition? Clin Neurophysiol. 2016;127:1845-54.

28. Thibaut A, Bruno M-A, Ledoux D, Demertzi A, Laureys S. tDCS in patients with disorders of consciousness: Sham-controlled randomized double-blind study. Neurology. 2014;82:1112-8.

29. Thibaut A, Wannez S, Donneau A-F, Chatelle C, Gosseries O, Bruno M-A, et al. Controlled clinical trial of repeated prefrontal tDCS in patients with chronic minimally conscious state. Brain Inj. 2017;31:466-74. 30. Cortese Md, Riganello F, Arcuri F, Pugliese Me, Lucca Lf, Dolce G, et al. Coma recovery scale-r: variability in the disorder of consciousness. BMC Neurol. 2015;15:186.

31. Giacino JT, Kalmar K, Whyte J. The JFK Coma Recovery Scale-Revised: Measurement characteristics and diagnostic utility. Arch Phys Med Rehabil. 2004;85:2020-9.

Page 15/19 
32. Boggio PS, Nunes A, Rigonatti SP, Nitsche MA, Pascual-Leone A, Fregni F. Repeated sessions of noninvasive brain DC stimulation is associated with motor function improvement in stroke patients. 2007;:7.

33. McMillan T, Wilson L, Ponsford J, Levin H, Teasdale G, Bond M. The Glasgow Outcome Scale -40 years of application and refinement. Nat Rev Neurol. 2016;12:477-85.

34. Department of Health. Mental Capacity Act. 2005. 2005;:92.

35. Cruse D, Chennu S, Chatelle C, Bekinschtein TA, Fernández-Espejo D, Pickard JD, et al. Bedside detection of awareness in the vegetative state: a cohort study. The Lancet. 2011;378:2088-94.

36. Cruse D, Chennu S, Fernández-Espejo D, Payne WL, Young GB, Owen AM. Detecting Awareness in the Vegetative State: Electroencephalographic Evidence for Attempted Movements to Command. PLoS ONE. 2012;7:e49933.

37. Bekinschtein TA, Coleman MR, Niklison J, Pickard JD, Manes FF. Can electromyography objectively detect voluntary movement in disorders of consciousness? J Neurol Neurosurg Psychiatry. 2008;79:8268.

38. Lesenfants D, Habbal D, Chatelle C, Schnakers C, Laureys S, Noirhomme Q. Electromyographic decoding of response to command in disorders of consciousness. Neurology. 2016;87:2099-107. 39. Fernández-Espejo D, Soddu A, Cruse D, Palacios EM, Junque $C$, Vanhaudenhuyse $A$, et al. A role for the default mode network in the bases of disorders of consciousness. Ann Neurol. 2012;72:335-43. 40. Lant ND, Gonzalez-Lara LE, Owen AM, Fernández-Espejo D. Relationship between the anterior forebrain mesocircuit and the default mode network in the structural bases of disorders of consciousness. Neurolmage Clin. 2016;10:27-35.

41. Chennu S, Annen J, Wannez S, Thibaut A, Chatelle C, Cassol H, et al. Brain networks predict metabolism, diagnosis and prognosis at the bedside in disorders of consciousness. Brain.

2017;140:2120-32.

42. Nitsche MA, Cohen LG, Wassermann EM, Priori A, Lang N, Antal A, et al. Transcranial direct current stimulation: State of the art 2008. Brain Stimulat. 2008;1:206-23.

43. Antal A, Alekseichuk I, Bikson M, Brockmöller J, Brunoni AR, Chen R, et al. Low intensity transcranial electric stimulation: Safety, ethical, legal regulatory and application guidelines. Clin Neurophysiol. 2017;128:1774-809.

44. Woods AJ, Antal A, Bikson M, Boggio PS, Brunoni AR, Celnik P, et al. A technical guide to tDCS, and related non-invasive brain stimulation tools. Clin Neurophysiol. 2016;127:1031-48.

45. Thibaut A, Schiff N, Giacino J, Laureys S, Gosseries 0 . Therapeutic interventions in patients with prolonged disorders of consciousness. Lancet Neurol. 2019;18:600-14.

46. Martens G, Lejeune N, O'Brien AT, Fregni F, Martial C, Wannez S, et al. Randomized controlled trial of home-based 4-week tDCS in chronic minimally conscious state. Brain Stimulat. 2018;11:982-90.

47. Laakso I, Tanaka S, Koyama S, De Santis V, Hirata A. Inter-subject Variability in Electric Fields of Motor Cortical tDCS. Brain Stimulat. 2015;8:906-913.

48. Opitz A, Paulus W, Will S, Antunes A, Thielscher A. Determinants of the electric field during transcranial direct current stimulation. Neurolmage. 2015;109:140-150.

49. Graham DI, Adams JH, Murray LS, B. J. Neuropathology of the vegetative state after head injury. 
Neuropsychol Rehabil. 2005;15:198-213.

50. Elliott $L$, Walker $L$. Rehabilitation interventions for vegetative and minimally conscious patients. Neuropsychol Rehabil. 2005;15:480-93.

51. Fernández-Espejo D, Owen AM. Detecting awareness after severe brain injury. Nat Rev Neurosci. 2013;14:801-9.

52. Kondziella D, Friberg CK, Frokjaer VG, Fabricius M, Møller K. Preserved consciousness in vegetative and minimal conscious states: systematic review and meta-analysis. J Neurol Neurosurg Psychiatry. 2016;87:485-92.

53. Graham M, Weijer C, Peterson A, Naci L, Cruse D, Fernández-Espejo D, et al. Acknowledging awareness: informing families of individual research results for patients in the vegetative state. $J$ Med Ethics. 2015;41:534-8.

\section{Tables}

\begin{tabular}{|c|c|c|c|c|c|c|c|c|c|c|c|}
\hline \multirow[b]{3}{*}{ TIMEPOINT** } & Enrolment & Allocation & \multicolumn{9}{|c|}{ Post-allocation } \\
\hline & \multicolumn{2}{|c|}{ Both streams } & \multicolumn{5}{|c|}{ MRI stream } & \multicolumn{4}{|c|}{ EEG stream } \\
\hline & $-w_{1}$ & 0 & $w_{1}$ & $w_{2}$ & $w_{3}$ & $w_{4}$ & $w_{5}$ & $w_{1}$ & $\begin{array}{l}w_{2-} \\
4\end{array}$ & $M_{3}$ & $M_{6}$ \\
\hline \multicolumn{12}{|l|}{ ENROLMENT: } \\
\hline $\begin{array}{l}\text { Eligibility screen (including } \mathrm{MRI}^{*} \text { and } \\
\text { tDCS safety) }\end{array}$ & $\mathrm{X}$ & & $\mathrm{X}$ & & $\mathrm{X}$ & & $\mathrm{X}$ & $\mathrm{X}$ & $\mathrm{X}$ & & \\
\hline *MRI stream only & & & & & & & & & & & \\
\hline Informed consent & $\mathrm{X}$ & & & & & & & & & & \\
\hline $\begin{array}{l}\text { Allocation (order of stimulation } \\
\text { conditions) }\end{array}$ & & $\mathrm{X}$ & & & & & & & & & \\
\hline \multicolumn{12}{|l|}{ STIMULATION: } \\
\hline Stimulation condition 1 & & & $\mathrm{X}$ & & & & & $\mathrm{X}$ & & & \\
\hline Stimulation condition 2 & & & & & $\mathrm{X}$ & & & & $\mathrm{X}$ & & \\
\hline Stimulation condition 3 & & & & & & & $\mathrm{X}$ & & & & \\
\hline \multicolumn{12}{|l|}{ ASSESSMENTS: } \\
\hline$C R S-R$ & $\mathrm{X}$ & $\mathrm{X}$ & $\mathrm{X}$ & & $\mathrm{X}$ & & $\mathrm{X}$ & $\mathrm{X}$ & $\mathrm{X}$ & & \\
\hline$M R I$ & & & $\mathrm{X}$ & & $\mathrm{X}$ & & $\mathrm{X}$ & & & & \\
\hline$E E G$ / EMG & & & $\mathrm{X}$ & & $\mathrm{X}$ & & $\mathrm{X}$ & $\mathrm{X}$ & $\mathrm{X}$ & & \\
\hline Behavioural command following & & & $\mathrm{X}$ & & $\mathrm{X}$ & & $\mathrm{X}$ & $\mathrm{X}$ & $\mathrm{X}$ & & \\
\hline GOSE & & & & & & & & & & $\mathrm{X}$ & $\mathrm{X}$ \\
\hline
\end{tabular}


Table 1. Summary of Interventions and Assessments.

\begin{tabular}{|l|l|}
\hline Variable & \\
\hline Eligibility & Percentage of participants screened deemed eligible \\
\hline Recruitment & Percentage of eligible participants willing to participate \\
\hline Retention & Pumber of participants actually recruited \\
\hline Completion & $\begin{array}{l}\text { Percentage of participants still on study after last tDCS intervention } \\
\text { Percentage of participants partially and fully active at 6-month follow-up }{ }^{\phi}\end{array}$ \\
\hline & $\begin{array}{l}\text { Percentage of MRI sessions completed per polarity* } \\
\text { Percentage of EEG/EMG sessions completed per polarity }\end{array}$ \\
\hline
\end{tabular}

\section{Table 2. Feasibility criteria}

*MRI stream only

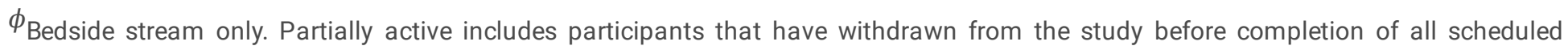
stimulation sessions but continue involvement for follow-up assessments. Fully active includes participants who have not withdrawn at any point

\section{Figures}




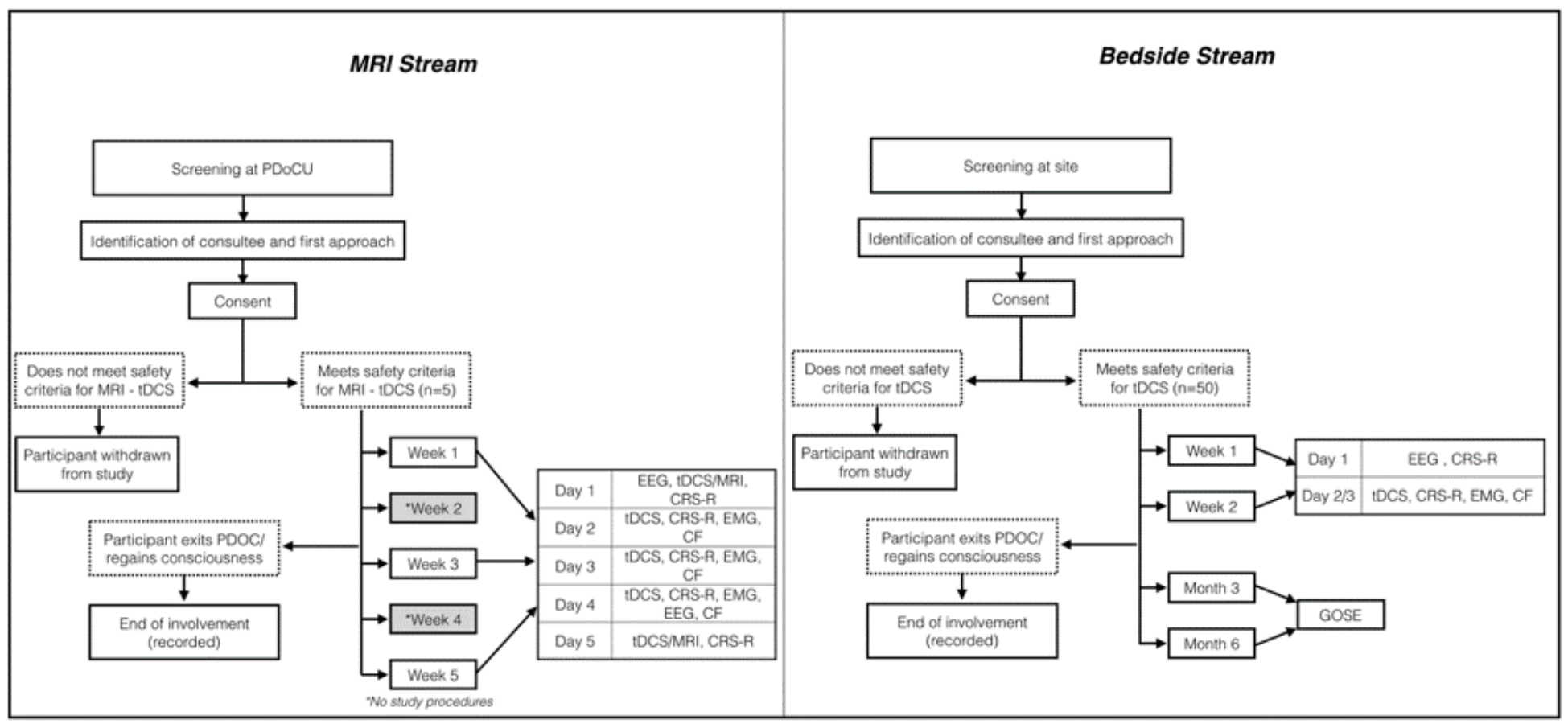

Figure 1

Study flowchart CF: command following; CRS-R: coma recovery scale-revised; EEG:

electroencephalography; EMG: electromyography; MRI: magnetic resonance imaging; tDCS: transcranial direct current stimulation; PDOC: prolonged disorders of consciousness; PDoCU: Prolonged disorders of consciousness Unit at the Wellington Hospital. 